OPEN ACCESS

Edited by:

Hiroyuki Ito,

Kyushu University, Japan

Reviewed by:

Wataru Teramoto,

Muroran Institute of Technology,

Japan

Behrang Keshavarz,

Toronto Rehabilitation Institute,

Canada

${ }^{*}$ Correspondence:

Shigehito Tanahashi,

Graduate School of Science and

Technology, Niigata University, 8050

Ikarashi 2 no-cho, Nishi-ku,

Niigata, Japan

tanahashi@eng.niigata-u.ac.jp

Specialty section:

This article was submitted to

Perception Science,

a section of the journal

Frontiers in Psychology

Received: 10 September 2014 Accepted: 20 May 2015

Published: 11 June 2015

Citation:

Tanahashi S, Ashihara $\mathrm{K}$ and Ujike $\mathrm{H}$ (2015) Effects of auditory information on self-motion perception during simultaneous presentation of visual shearing motion.

Front. Psychol. 6:749.

doi: 10.3389/fpsyg.2015.00749

\section{Effects of auditory information on self-motion perception during simultaneous presentation of visual shearing motion}

\author{
Shigehito Tanahashi ${ }^{*}$ Kaoru Ashihara and Hiroyasu Ujike \\ Sensory and Perceptual Information Design Group, Human Informatics Research Institute, National Institute of Advanced \\ Industrial Science and Technology, Tsukuba, Japan
}

Recent studies have found that self-motion perception induced by simultaneous presentation of visual and auditory motion is facilitated when the directions of visual and auditory motion stimuli are identical. They did not, however, examine possible contributions of auditory motion information for determining direction of self-motion perception. To examine this, a visual stimulus projected on a hemisphere screen and an auditory stimulus presented through headphones were presented separately or simultaneously, depending on experimental conditions. The participant continuously indicated the direction and strength of self-motion during the 130-s experimental trial. When the visual stimulus with a horizontal shearing rotation and the auditory stimulus with a horizontal one-directional rotation were presented simultaneously, the duration and strength of self-motion perceived in the opposite direction of the auditory rotation stimulus were significantly longer and stronger than those perceived in the same direction of the auditory rotation stimulus. However, the auditory stimulus alone could not sufficiently induce self-motion perception, and if it did, its direction was not consistent within each experimental trial. We concluded that auditory motion information can determine perceived direction of self-motion during simultaneous presentation of visual and auditory motion information, at least when visual stimuli moved in opposing directions (around the yaw-axis). We speculate that the contribution of auditory information depends on the plausibility and information balance of visual and auditory information.

Keywords: self-motion, vection, vision, audition, subjective score, random dot pattern, pink noise, perception

\section{Introduction}

The present study focused on the effect of auditory information presented with visual information on self-motion perception. When we operate in dynamic environments, self-motion information can be obtained from the visual and auditory systems, as well as the vestibular, somatosensory, and proprioceptive systems (Lishman and Lee, 1973; Dichgans and Brandt, 1978; Warren and Wertheim, 1990; DeAngelis and Angelaki, 2012). Visual self-motion information is provided by visual global motion, or more specifically, optic flow (Gibson, 1961), and auditory self-motion information is provided by loudness changes, binaural cues, and the Doppler effect of sound (Lutfi and Wang, 1999). 
Most previous studies have examined visual and auditory perception of self-motion independently. Self-motion perception induced by visual motion alone is a well-known phenomenon that has been recognized since at least the 19th century (Mach, 1875; Wood, 1895). This phenomenon was named vection by Fisher and Kommüler (1930) (for an overview, see Hettinger et al., 2014). Similar to visually induced self-motion, auditorily induced self-motion has long been recognized (Urbantschtsch, 1897; Dodge, 1923). For example, observers wearing eye masks perceive self-motion through auditory motion alone (Lackner, 1977; Larsson et al., 2004; Sakamoto et al., 2004; Väljamäe et al., 2004; Riecke et al., 2009; Väljamäe, 2009). Visually or auditorily induced self-motion is typically perceived in the opposite direction of visual or auditory motion, respectively.

Recently, several studies have examined the effect of visual and auditory inputs in self-motion perception by simultaneously presenting visual and auditory motion stimuli. Riecke et al. (2009), Seno et al. (2012), and Keshavarz et al. (2014) compared the magnitude of self-motion perception obtained by a visual motion stimulus alone and that obtained by simultaneous presentation of visual and auditory motion stimuli, finding that self-motion perception is facilitated when the directions of visual and auditory motion stimuli are identical. Moreover, Seno et al. (2012) reported that self-motion perception is neither inhibited nor facilitated by directional conflict between visual and auditory motion stimuli, while perceived linear direction is determined by the visual motion stimulus. These results indicate that self-motion perception can be facilitated with simultaneous presentation of visual and auditory stimuli, while visual information determines the direction of self-motion perception. However, this study did not fully examine whether directional information from auditory stimuli contributes to selfmotion perception during simultaneous presentation of visual and auditory stimuli.

We investigated the possible contribution of directional information from an auditory stimulus on rotational selfmotion perception during simultaneous presentation of visual and auditory rotational stimuli. To this end, we adopted visual rotational shearing motion so that visual information did not determine the direction of self-motion perception (for vertical linear shearing motion, see Kitazaki and Sato, 2003). If directional information of auditory stimuli can be used, perceived rotational direction is expected to be opposite to auditory rotation during simultaneous presentation of visual shearing motion and auditory rotation stimuli. Moreover, the visual and auditory stimuli we used were rotated around the vertical yaw axis based on previous findings (Toshima et al., 2006, 2008), which indicated that the accuracy of sound localization on the horizontal plane is greater than that on the median plane. Considering these results, greater accuracy of sound localization was expected to more easily produce self-motion perception from auditory rotation stimuli.

\section{Materials and Methods}

\section{Apparatus}

We used an LCD projector (Epson ELP-7700) with a fisheye lens to project a visual stimulus onto the inside of a hemisphere (with a 150-cm inner diameter; Figure 1A). Headphones (BOSE TP1SB TriPort) were used to present an auditory stimulus. We installed a chair in front of the hemisphere screen surface so that an observer sitting upright had her/his feet not touching the floor. With this installation, we expected that the observer would more easily perceive self-motion by her-/himself (Lepecq et al., 1995; Wright et al., 2006; Riecke et al., 2008).

\section{Stimuli}

\section{Visual Stimuli}

A visual stimulus that simulated a sphere with a $150-\mathrm{cm}$ diameter (Figure 1A) was rendered in real time as a computer-generated image on a Windows-based PC (Dual Core 2, $2.4 \mathrm{GHz}$ ) with OpenGL. The frame rate was $60 \mathrm{~Hz}$, and the image size was a diameter of about $94^{\circ}$ from a viewing distance of about $70 \mathrm{~cm}$; the vantage point was the center of perspective projection. The rendered visual image was a random dot pattern consisting of white dots (diameter of dot: $1.39^{\circ}$; luminance: $3.03 \mathrm{~cd} / \mathrm{m}^{2}$ ) on a black background $\left(0.60 \mathrm{~cd} / \mathrm{m}^{2}\right)$ that was generated on the inner surface of the sphere. The white dots accounted for $33 \%$ of the area of the inner surface of the sphere. The fixation point (a red dot; diameter of dot: $2.46^{\circ}$; luminance: $1.05 \mathrm{~cd} / \mathrm{m}^{2}$ ) was presented on the center of the inner surface of the sphere. The visual stimulus simulated rotation of the observer around the yaw axis. Both directions of rotation around the yaw axis were used. The rotation velocity was held constant at $36^{\circ} \%$. The velocity, $36^{\circ} / \mathrm{s}$, was determined based on especially the auditory condition that tends to produce self-motion perception. Actually, the previous studies that examined auditorily induced self-motion perception used a stimulus velocity of $30^{\circ} \%$ s (Riecke et al., 2009) and $40^{\circ} / \mathrm{s}$ (Larsson et al., 2004). The specific value, $36^{\circ} / \mathrm{s}$, was chosen in order to produce a stimulus between the values used in previous studies.

We used four different types of visual stimulus condition. The first was a no-visual-stimulus (V_NRD) condition, in which the observer wore an eye mask. In the other three conditions, three different moving patterns of random dots were presented: (i) stationary (V_SRD; Figure 1B), (ii) horizontal one-directional motion (V_HOM; Figure 1C), and (iii) horizontal shearing motion between vertically adjacent stripe-shaped areas (V_HSM; Figure 1D). The horizontal shearing motion was produced by six vertically adjacent striped areas, each of which used random dot motion in opposite directions relative to each other; the striped areas had a width of approximately $15.7^{\circ}$, and no visual border line between the adjacent areas.

\section{Auditory Stimuli}

The auditory stimulus was a pink noise presented through headphones. We adopted this auditorily abstract stimulus corresponding to visual random dot stimulus. A-weighted sound pressure level for the observer was $64 \mathrm{~dB}$. Both rotation directions around the yaw axis were used. The rotational auditory stimulus was produced with the following processes. First, a pink noise was synthesized and generated by two notebook computers presented from three loudspeakers (Maxer-denki MSW-SB) located at an equal distance and an equal angle from the rotating base (SHIMPO RK-5T). Second, this auditory stimulus was recorded by a dummy head with a binaural microphone (Audio-Technica 


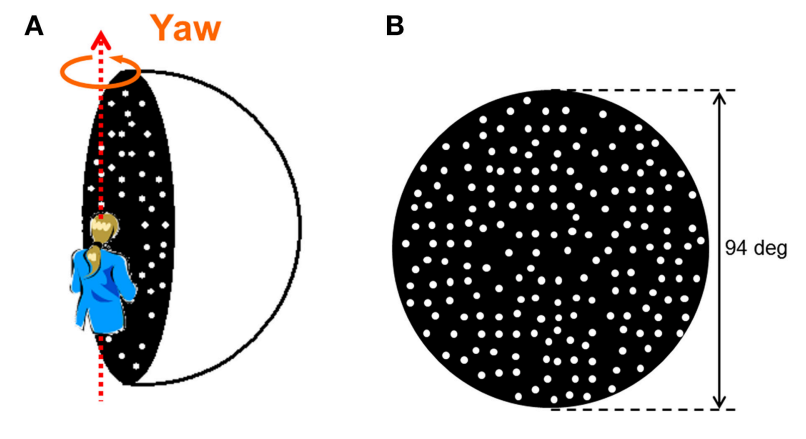

FIGURE 1 | Visual stimulus on the inside of the hemisphere screen. (A) The random dot pattern that was rotated along the yaw axis relative to the observer was projected onto the inside of a hemisphere screen. In this experiment, the participant sat in
C

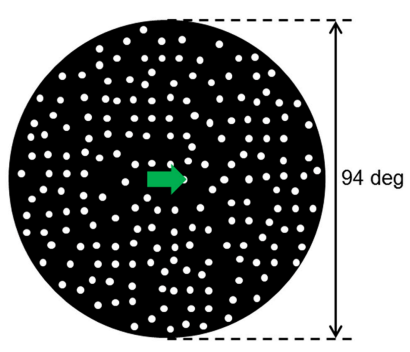

D

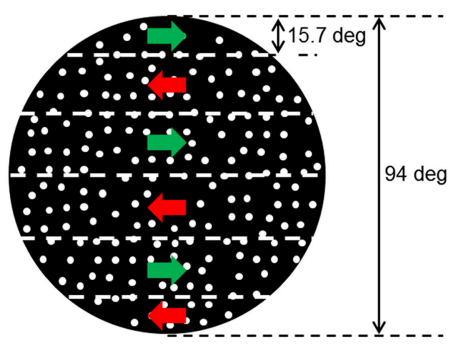

an upright position; (B) stationary random dot pattern (V_SRD); (C) horizontal one-directional motion (V_HOM); and (D) horizontal shearing motion between vertically adjacent stripe-shaped areas (V_HSM).

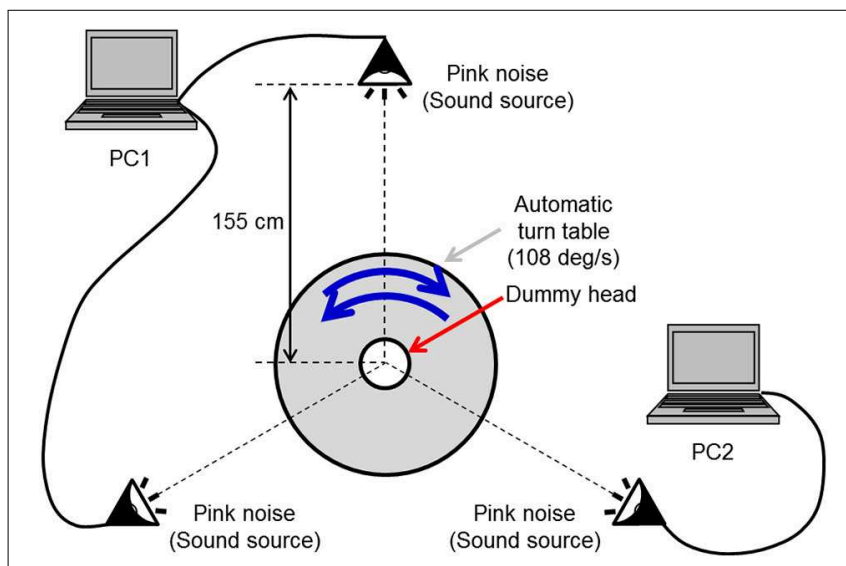

FIGURE 2 | The rotational auditory stimulus. A pink noise was output by two notebook computers through three loudspeakers located at an equal distance and an equal angle from the rotating base. This auditory stimulus was recorded by a dummy head with a binaural microphone.

AT9903; Figure 2). This dummy head was installed on the rotation center of the rotating base, and it was one-third the size of an averaged normal human head. To equalize a frequency characteristic of this dummy head to that of a normal dummy head, we converted the rotational velocity of the rotating base and the pitch of the recorded auditory stimulus. The velocity was constant at $108^{\circ} / \mathrm{s}$ and the pitch was one-third of the original pink noise. Then, the recorded sound was played at one-third normal speed so that the rotational velocity of the auditory stimulus became constant at $36^{\circ} \%$, identical to that of the visual stimulus.

We used three different types of auditory stimulus condition. The first was the no-auditory-stimulus (A_NPN) condition. In the other two conditions, two different moving patterns of the pink noise were presented: (i) stationary (A_SPN) and (ii) horizontal one-directional motion simulating rotation of the sound source around the yaw axis (A_HOM).

\section{Procedure}

After providing informed written consent, participants were explained and experienced the self-motion perception induced by visual and auditory rotational stimulus alone. The explanation of the perception was given as an experience that participants would perceive as though their own body was rotated around an axis. Then, participants were exposed to a horizontal one-directional visual and auditory motion stimuli, such as in V_HOM + A_NPN or V_NRD + A_HOM conditions, for $1 \mathrm{~min}$. After the exposure, all the participants reported that they perceived visually induced self-motion in the V_HOM + A_NPN condition. However, not all participants reported perceiving auditorily induced self-motion in the V_NRD + A_HOM condition.

Before each trial begun, participants were adapted to darkness for $10 \mathrm{~min}$ by sitting in a quasi-dark room while wearing an eye mask. After the adaptation, the participant wore headphones, and a 130-s experimental trial begun. At the beginning of each trial, a stationary image without an auditory stimulus was presented for $10 \mathrm{~s}$, followed by a stimulus combining visual and auditory stimuli for $120 \mathrm{~s}$.

The stimulus conditions were combinations of the four visual stimulus conditions and the three auditory stimulus conditions. However, we excluded three of the above combinations, V_NRD + A_NPN, V_NRD + A_SPN, and V_SRD + A_NPN, resulting in nine different experimental conditions (Table 1). The sign "+" here represents the combination of visual and auditory stimulus conditions.

There were 22 experimental trials for each observer; these trials were performed in a randomized order across participants. The detailed trial numbers for each experimental condition are presented in Table 1. These were determined as follows: (a) two trials were assigned to V_HOM and V_HSM as well as A_HOM, corresponding to the two directional rotations of those stimulus conditions, and (b) two trials were assigned to the experimental condition V_SRD + A_SPN. Because of (a), four trials were performed in both $\mathrm{V} \_\mathrm{HOM}+\mathrm{A} \_\mathrm{HOM}$ and V_HSM + A_HOM, corresponding to the combinations of two directional rotations of both visual and auditory stimulus conditions.

To measure the direction and strength of self-motion perception, we adopted a continuous subjective measurement during each trial. The continuous measurements were adopted in accordance with previous studies showing that observers 


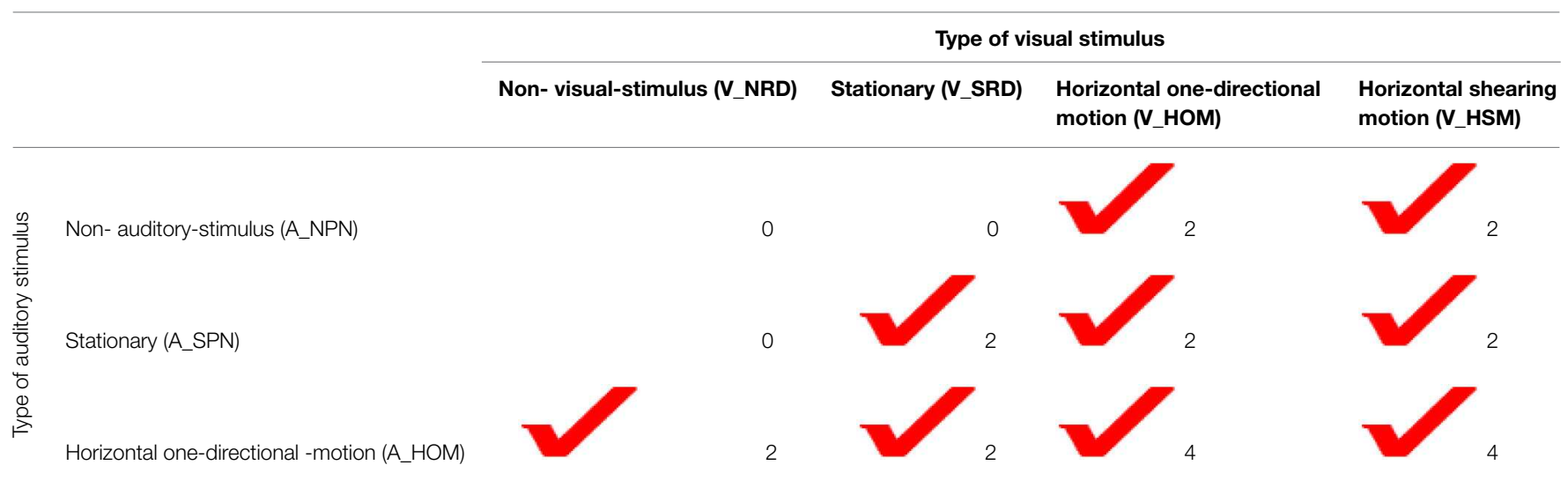

The bottom-right number in each cell is the detailed trial number for each experimental condition.

TABLE 2 | Detailed 7-point scale of the change in direction and strength of self-motion perception.

\begin{tabular}{lcl}
\hline Clockwise direction & +3 & The perceived self-motion could not be \\
around the yaw axis & +2 & differentiated from real physical motion \\
& +1 & \\
& 0 & No self-motion was perceived \\
Counterclockwise & -1 & \\
direction around the yaw & -2 & \\
axis & -3 & The perceived self-motion could not be \\
& & differentiated from real physical motion
\end{tabular}

often experienced intermittent self-motion perception induced by visual motion stimuli (Kleinschmidt et al., 2002; Tanahashi et al., 2012). During a trial, whenever the observers experienced self-motion perception, they continuously indicated the change in the direction and strength of self-motion perception using a subjective response box to evaluate perception on a 7-point scale (Table 2). In Table 2, “+” represents self-motion in a clockwise (CW) direction around the yaw axis, and “-” represents selfmotion perceived in a counterclockwise (CCW) direction around the yaw axis. The value " 0 " indicates that no self-motion was perceived, and " 3 " indicates that the perceived self-motion could not be differentiated from real physical motion. Participants were able to clearly indicate the value of their self-motion perception without looking at the subjective response box because a gap, which was tactually perceived, was provided for each self-motion perception value on a linear potentiometer. These data were recorded at a sampling rate of $60 \mathrm{~Hz}$.

Upon completion of these tasks, each participant rested for $10 \mathrm{~min}$ in the quasi-dark room before advancing to the next trial. All participants completed 11 trials per day across two separate days.

\section{Participants}

Ten adults (seven women and three men; $34.4 \pm 8.15$ years) participated in the study after providing informed written consent, in accordance with the provisions of the Ergonomics
Experiment Policy of the National Institute of Advanced Industrial Science and Technology (AIST). The observers were free to withdraw at any time during the experiment. The experimental protocol was approved in advance by the Institutional Review Board of AIST. The observers were naïve as to the purpose of the experiment, and had normal or correctedto-normal visual acuity and normal hearing. Visual acuity was tested using the standard optotype of Landolt rings at $5 \mathrm{~m}$ and another optotype at $30 \mathrm{~cm}$. Stereo acuity was tested using the stereo test chart (Stereo Optical Randot ${ }^{\circledR}$ Stereotest). Standard pure-tone audiometry was conducted using an audiometer (RION AA-58). Participants did not have eye or ear disease.

\section{Results}

We examined the effect of auditory information presented with visual information on self-motion perception using the three resulting values: the strength, duration, and onset latency of self-motion perception. These values were extracted from the temporal variations in self-motion strength reported by participants. For each experimental trial, strength was averaged and duration was summed for each direction of self-motion perception. Onset latency was obtained using the time from the onset of visual and auditory stimulus presentation to the first onset of self-motion perception. When the observers did not perceive self-motion, the value of onset latency of selfmotion perception was excluded from averaging and other statistical calculation. These three values were each averaged across participants.

When the visual stimulus of stationary random dot patterns and the auditory stimulus of stationary pink noise were presented simultaneously (V_SRD + A_SPN), none of the participants perceived self-motion in any of the experimental trials (zero out of 20 trials).

\section{When Visual Stimulus Moved in Opposing Directions (Shearing Motion)}

When the visual stimulus with a horizontal shearing motion and no auditory stimulus was presented (V_HSM + A_NPN), 


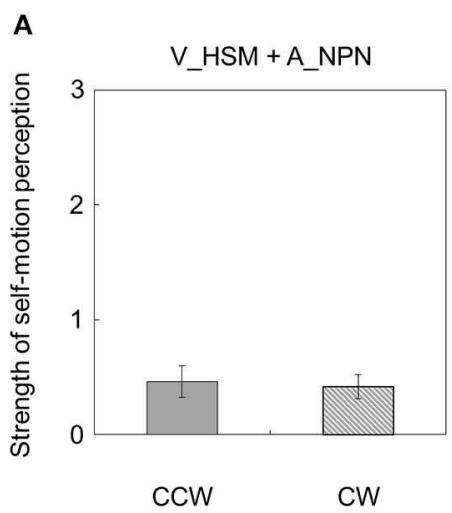

Perceived direction of self-motion

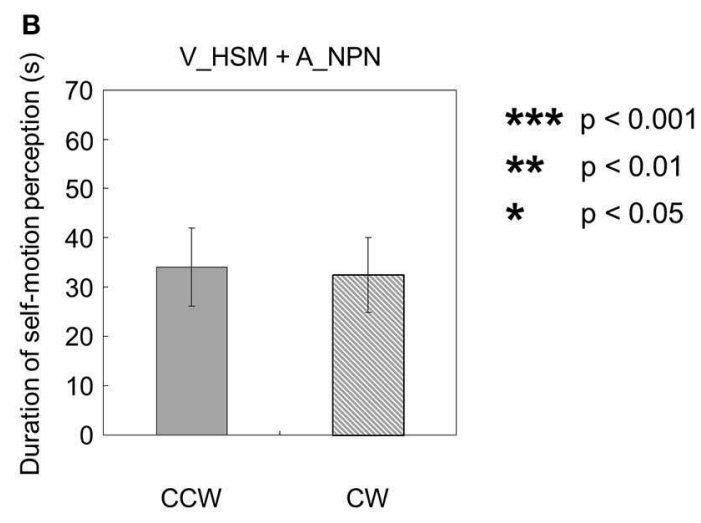

Perceived direction of self-motion
FIGURE 3 | Averaged values of the (A) strength and (B) duration of self-motion perception in the condition combining visually horizontal shearing motion (V_HSM) and auditorily horizontal one-directional motion (A_HOM). The values are plotted by clockwise (CW) and counterclockwise (CCW) directions. The error bars represent standard error (SE).

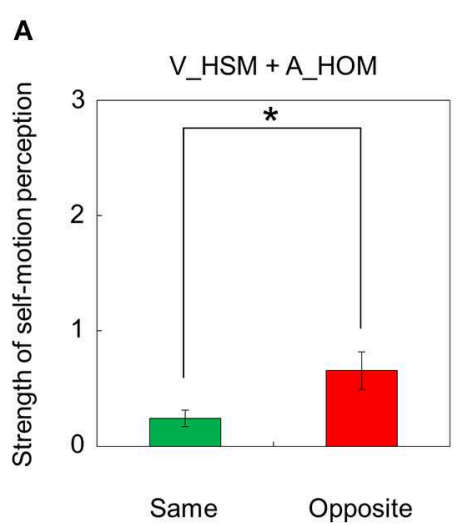

Directional consistency of self-motion perception and auditory rotation stimulus

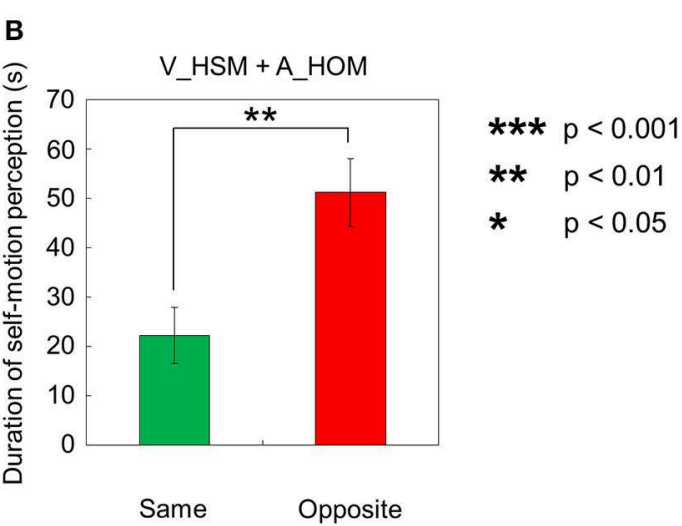

Directional consistency of self-motion perception and auditory rotation stimulus
FIGURE 4 | Averaged values of the (A) strength and (B) duration of self-motion perception in the condition combining visually horizontal shearing motion (V_HSM) and no-auditory-stimulus
(A_NPN). The values are plotted by the directional consistency of self-motion perception and the auditory rotation stimulus. The error bars represent standard error (SE). self-motion was perceived in both rotation directions around the yaw axis (Figures 3A,B). The strength of self-motion was not significantly different regardless of whether self-motion perception was CW or CCW $\left[t_{(9)}=0.025, p=0.98, d=0.011\right]$. Moreover, the duration of self-motion episodes in each stimulus condition was not significantly different across directions of self-motion perception $\left[t_{(9)}=0.41, p=0.69, d=0.22\right]$.

However, when the visual stimulus with a horizontal shearing motion and the auditory stimulus with a horizontal onedirectional motion were presented simultaneously (V_HSM + A_HOM), self-motion was perceived stronger and longer in the opposite direction of the auditory rotation stimulus (Figures 4A,B). The strength and duration of self-motion that was perceived in the opposite direction of the auditory rotation stimulus were significantly stronger and longer than those perceived in the same direction as the auditory rotation stimulus [strength: $t_{(9)}=2.38, p=0.041, d=0.99$; duration: $t_{(9)}=2.83$, $p=0.02, d=1.37$.

Moreover, when the visual stimulus with a horizontal shearing motion and the auditory stimulus with a stationary pink noise were presented simultaneously (V_HSM + A_SPN), the strength and duration of self-motion were not significantly different, regardless of the direction of the self-motion perception [strength: $t_{(9)}=0.23, p=0.82, d=0.11$; duration: $t_{(9)}=0.11$, $p=0.91, d=0.060$; Figures 5A,B].

The onset latency of self-motion perception were compared among the above three experimental conditions (V_HSM + A_NPN vs. V_HSM + A_HOM vs. V_HSM + A_SPN). To do this, we averaged the values of onset latency across observers for each experimental condition. We found no significant differences using a One-Way repeated measures ANOVA [onset latency: $F_{(2,27)}=1.78, p=0.19, \widehat{\eta}^{2}=0.12$; Figures 6A-C] 


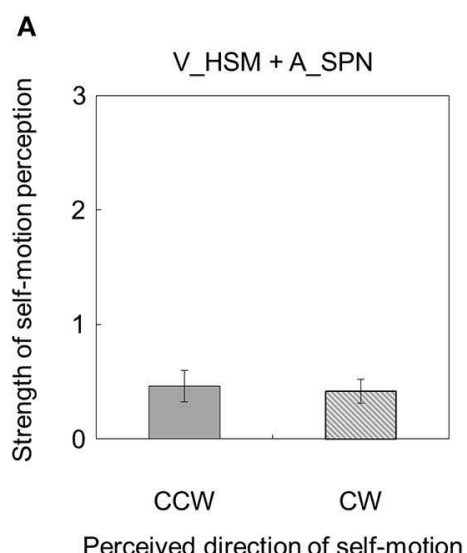

FIGURE 5 | Averaged values of the (A) strength and (B) duration of self-motion perception in the condition combining visually horizontal shearing motion (V_HSM) and auditorily stationary

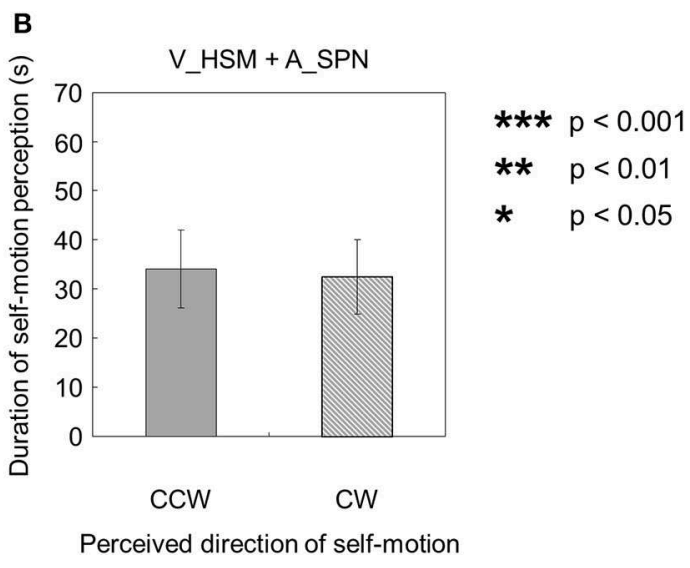

pink noise (A_SPN). The values are plotted by clockwise (CW) and counterclockwise (CCW) directions. The error bars represent standard error (SE).

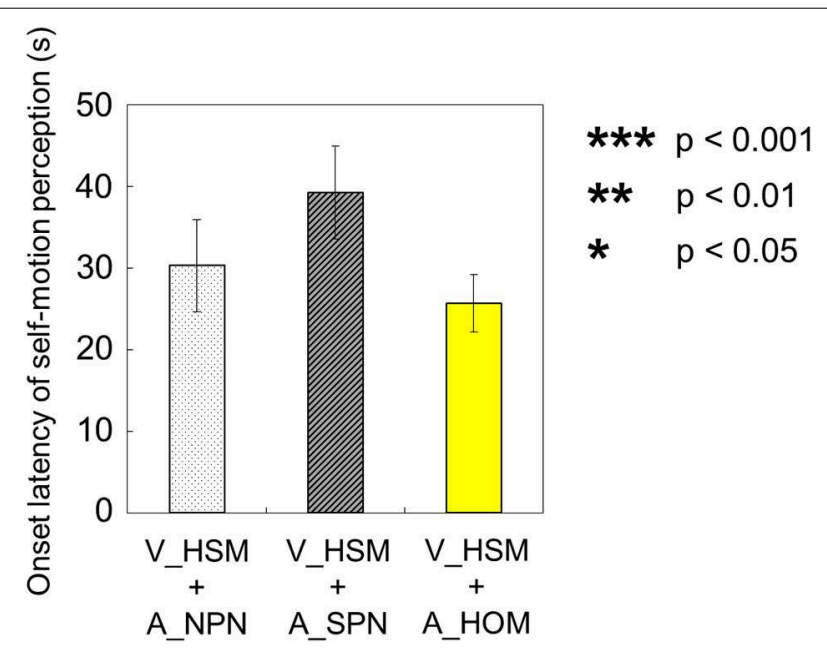

FIGURE 6 | Averaged values of the onset latency of self-motion perception in the three experimental conditions including visual shearing motion. In each graph, the left bar shows the condition combining visually horizontal shearing motion (V_HSM) and no auditory stimulus (A_NPN). The middle bar is the combination of visual horizontal shearing motion between the vertically adjacent stripe-shaped area (V_HSM) and stationary pink noise (A_SPN). The right bar is the combination of the vertically adjacent stripe-shaped area (V_HSM) and horizontal one-directional motion simulating rotation of pink noise around the yaw axis (A_HOM). The error bars represent standard error (SE).

All participants perceived self-motion among the above three experimental conditions.

\section{When Either the Visual or the Auditory Stimulus Moved in One Direction}

When either the visual or auditory stimulus was rotated horizontally in a one-directional motion without the counterpart of the stimulus (visual stimulus alone: V_HOM + A_NPN; auditory stimulus alone: V_NRD + A_HOM), participants tended to perceive self-motion. First, for the auditory stimulus with no visual stimulus (V_NRD + A_HOM), five participants perceived self-motion. The duration and strength of auditorily induced self-motion in opposite direction of the auditory rotation stimulus was significantly greater than zero [strength: $t_{(9)}=2.45, p=0.036, d=1.16$; duration: $t_{(9)}=2.45$, $p=0.037, d=1.23$ ]. Among them, three participants always perceived self-motion in the opposite direction of the auditory rotation stimulus; the other two did not always perceive in the opposite direction of the auditory rotation stimulus throughout the trials. The duration and strength of auditorily induced selfmotion were not significantly different, regardless of the direction of self-motion perception [strength: $t_{(9)}=1.33, p=0.22, d=$ 0.58 ; duration: $t_{(9)}=1.75, p=0.11, d=0.39$; Figures 7A,B].

Second, for the visual stimulus with no auditory stimulus (V_HOM + A_NPN), all participants perceived self-motion. Among them, nine participants always perceived self-motion in the opposite direction of the auditory rotation stimulus; the other one did not always perceive in the opposite direction of the auditory rotation stimulus throughout the trials. In this experimental condition, the strength and duration of visually induced self-motion that was perceived in the opposite direction of the visual rotation stimulus were significantly longer and stronger than those perceived in the same direction as the visual rotation stimulus [strength: $t_{(9)}=3.45, p=$ $0.0073, d=1.91$; duration: $t_{(9)}=6.00, p=0.0011$, $d=2.82$; Figures 8A,B]. Moreover, we compared the strength and duration of perceived self-motion across the above two experimental conditions. To this end, we averaged the amount of time that the participants experienced self-motion in the opposite direction of visual/auditory motion and the mean strength of perceived self-motion during the time, and averaged each of the above values across trials by either the visual or auditory information. We then found that the strength and duration of visually induced self-motion were stronger and longer than those of auditorily induced self-motion [strength: $t_{(9)}=4.20, p<$ $0.001, d=1.98$; duration: $t_{(9)}=4.51, p=0.0015, d=$ 


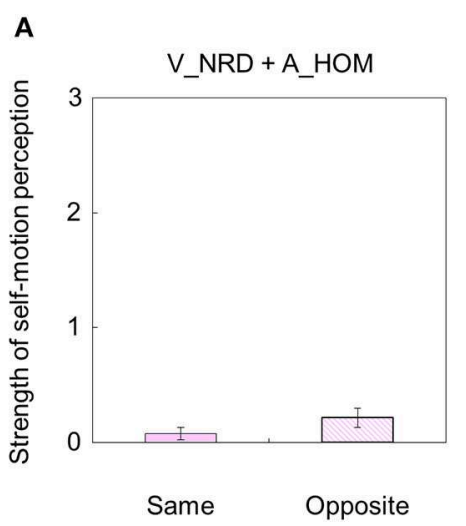

Directional consistency of self-motion perception and auditory rotation stimulus

FIGURE 7 | Averaged values of the (A) strength and (B) duration of self-motion perception in the condition combining no visual stimulus (V_NRD) and auditory horizontal one-directional motion
B

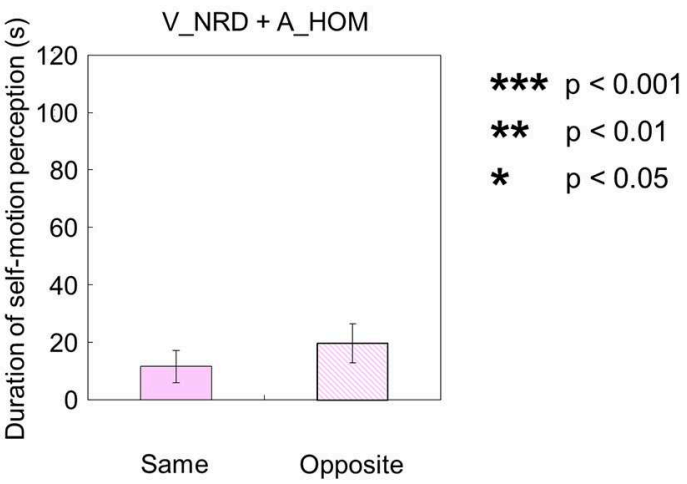

Directional consistency of self-motion

perception and auditory rotation stimulus

(A_HOM). The values are plotted the directional consistency of self-motion perception and auditory rotation stimulus. The error bars represent standard error (SE).

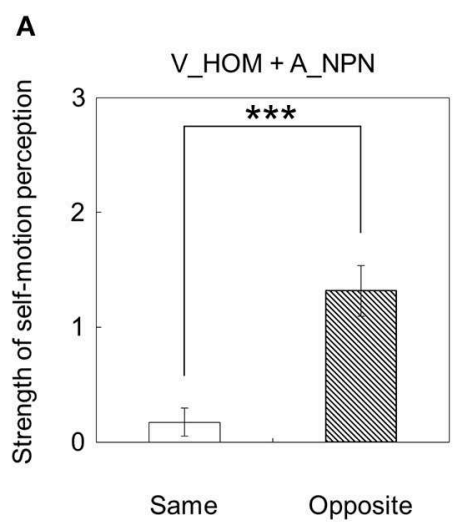

Directional consistency of self-motion perception and visual rotation stimulus

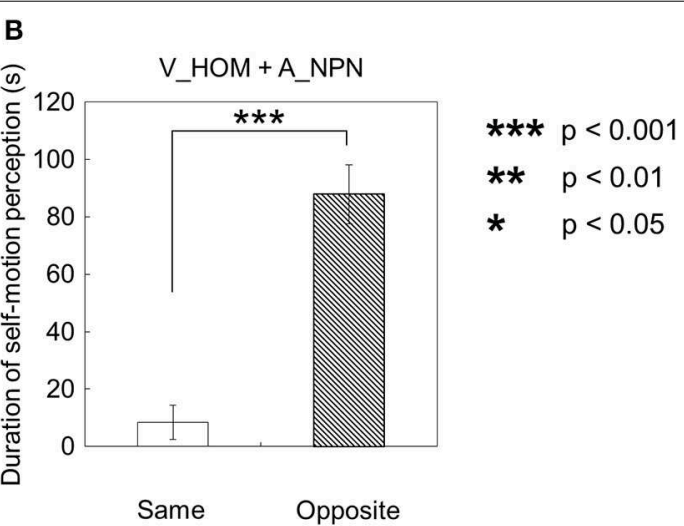

Directional consistency of self-motion perception and visual rotation stimulus
FIGURE 8 | Averaged values of (A) strength and (B) duration of self-motion perception in the condition combining visual horizontal one-directional motion (V_HOM) and no-auditory-stimulus (A_NPN) conditions. The values are plotted by separating the directional consistency of self-motion perception and the visual rotation stimulus. The error bars represent standard error (SE).
1.80; Figures 9A,B]. However, the onset latency of self-motion was not significantly different regardless of whether self-motion perception was induced by the visual or the auditory stimulus, which is indicated by the paired-samples $t$-tests using the data of participants who experienced self-motion perception in both conditions $\left[{ }^{1} t_{(4)}=1.55, p=0.20, d=0.47\right.$; Figure 9C].

Similarly, when either the visual or auditory stimulus was rotated horizontally in a one-directional motion with the stationary stimulus of the counterpart (auditory rotation stimulus with visual stationary stimulus: V_SRD + A_HOM; visual rotation stimulus with auditory stationary stimulus:

\footnotetext{
${ }^{1}$ When we compared the onset latency of perceived self-motion across the V_NRD + A_HOM and V_HOM and A_NPN conditions using unpaired-samples $t$ tests, the onset latency of self-motion was not significantly different regardless of whether self-motion perception was induced by the visual or the auditory stimulus $\left[t_{(13)}=0.82, p=0.43, d=0.48\right]$.
}

V_HOM + A_SPN), participants tended to perceive self-motion. First, for the auditory stimulus with stationary visual stimulus (V_SRD + A_HOM), six participants perceived self-motion. Among them, four participants always perceived self-motion in the opposite direction of the auditory rotation stimulus; the other two did not always perceive in the opposite direction of the auditory rotation stimulus throughout the trials. The duration and strength of auditorily induced self-motion in opposite direction of the auditory rotation stimulus was not significantly greater than zero [strength: $t_{(9)}=1.42, p=0.19$, $d=0.64$; duration: $\left.t_{(9)}=1.42, p=0.19, d=0.64\right]$. The duration and strength of auditorily induced self-motion were not significantly different, regardless of the direction of selfmotion perception [strength: $t_{(9)}=1.54, p=0.16, d=0.46$ duration: $t_{(9)}=1.37, p=0.20, d=0.42$ ]. Second, for the visual stimulus with stationary auditory stimulus (V_HOM + 


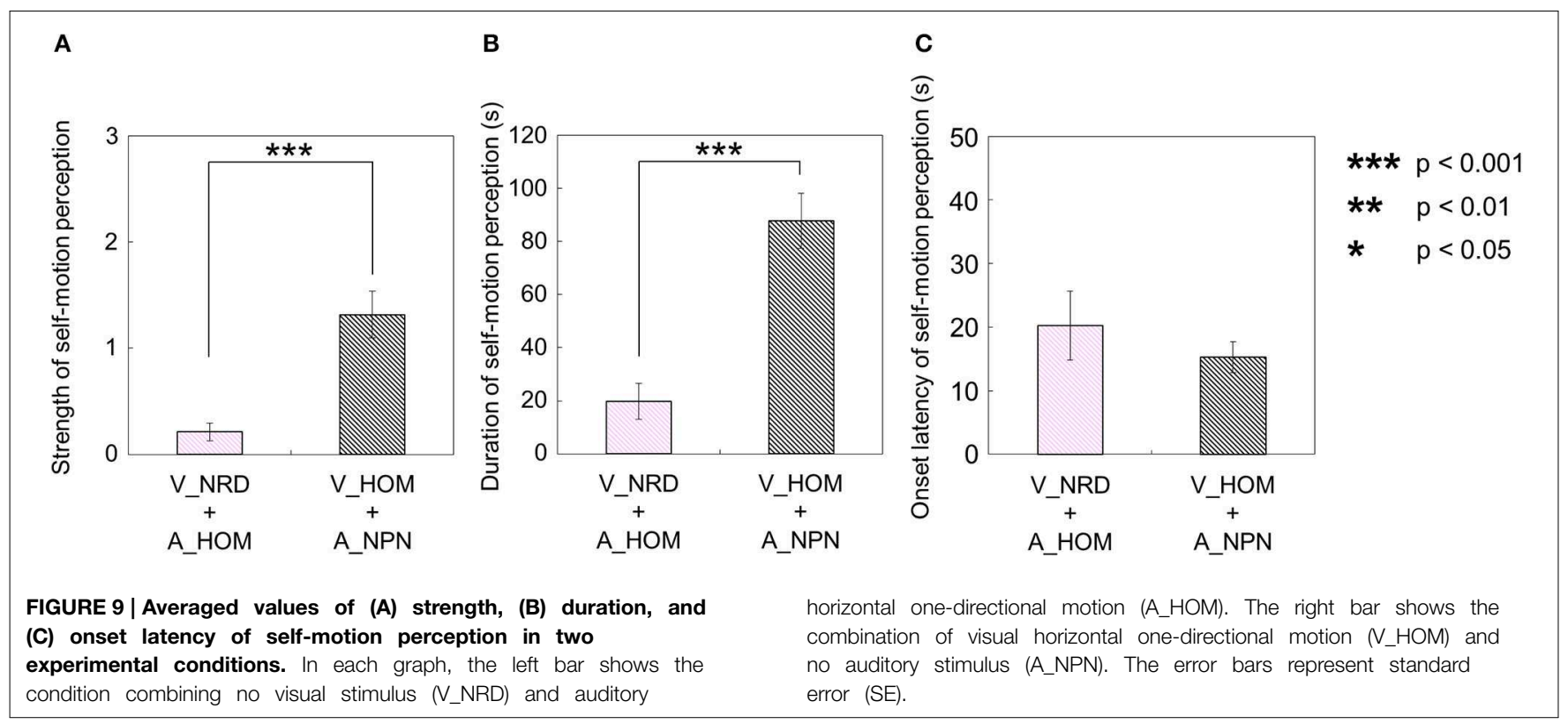

A_SPN), all participants perceived self-motion. Among them, seven participants always perceived self-motion in the opposite direction of the auditory rotation stimulus; the other three did not always perceive in the opposite direction of the auditory rotation stimulus throughout the trials. In this experimental condition, the strength and duration of visually induced selfmotion that was perceived in the opposite direction of the visual rotation stimulus were significantly longer and stronger than those perceived in the same direction as the visual rotation stimulus [strength: $t_{(9)}=6.55, p<0.001, d=2.16$; duration: $\left.t_{(9)}=9.00, p<0.001, d=3.15\right]$. Moreover, we compared the strength and duration of perceived self-motion across the above two experimental conditions. To this end, we averaged the amount of time that the participants experienced self-motion in the opposite direction of visual/auditory motion and the mean strength of perceived self-motion during the time, and averaged each of the above values across trials by either the visual or auditory information. We then found that the strength and duration of visually induced self-motion were stronger and longer than those of auditorily induced self-motion [strength: $t_{(9)}=7.23, p<0.001, d=2.31$; duration: $t_{(9)}=11.6$ $p<0.001, d=3.47]$. However, the onset latency of self-motion was not significantly different regardless of whether self-motion perception was induced by the two experimental conditions above, which is indicated by the paired samples $t$-tests using the data of participants who experienced self-motion perception in both conditions $\left[{ }^{2} t_{(3)}=1.04, p=0.37, d=0.28\right]$.

To examine the effects of existence of stationary visual stimulus on auditory-induced self-motion perception, we

\footnotetext{
${ }^{2}$ When we compared the onset latency of perceived self-motion across the V_SRD + A_HOM and V_HOM and A_SPN conditions using unpaired-samples $t$-tests, the onset latency of self-motion was not significantly different regardless of whether self-motion perception was induced by the above two experimental conditions $[t(12)=2.08, p=0.060, d=1.33]$.
}

compared each of the three values-strength, duration, and onset latency-of self-motion perception across the two different experimental conditions (V_NRD + A_HOM vs. V_SRD + A_HOM). To do this, we averaged amount of time that the participants experienced self-motion in the opposite direction of visual/auditory motion and the mean strength of perceived self-motion during the time, and averaged each of the above values across trials by auditory information. We found no significant differences between the two experimental conditions using a paired-samples $t$-test [strength: $t_{(9)}=1.88, p=0.092$, $d=0.84$; duration: $t_{(9)}=1.97, p=0.081, d=0.82$; onset latency: ${ }^{3} t_{(2)}=0.028, p=0.98, d=0.0071$; Figures 10A-C].

Finally, to examine the effect of existence of visual rotational shearing motion on auditory-induced self-motion perception, we compared each of the three values - strength, durationof self-motion perception across the two different experimental conditions (V_NRD + A_HOM vs V_HSM + A_HOM). The strength and duration of self-motion perception in the V_HSM + A_HOM condition were stronger and longer than that in the V_NRD + A_HOM condition [strength: $\underline{t}_{(9)}=2.53, p=0.032$, $d=0.75$; duration: $\left.t_{(9)}=2.65, p=0.026, d=0.84\right]$. However, the onset latency of self-motion was not significantly different regardless of whether self-motion perception was induced by the above two experimental conditions, which is indicated by the paired samples $t$-tests using the data of participants who experienced self-motion perception in both conditions $\left[{ }^{4} t_{(4)}=\right.$ $0.43, p=0.69, d=0.096]$.

\footnotetext{
${ }^{3}$ When we compared the onset latency of perceived self-motion across the $\mathrm{V} \_\mathrm{NRD}+\mathrm{A} \_\mathrm{HOM}$ and $\mathrm{V} \_\mathrm{SRD}$ and A_HOM conditions using unpaired-samples $t$-tests, the onset latency of self-motion was not significantly different regardless of whether self-motion perception was induced by the above two experimental conditions $[t(7)=1.50, p=0.18, d=1.13]$.

${ }^{4}$ When we compared the onset latency of perceived self-motion across the $\mathrm{V} \_\mathrm{NRD}+\mathrm{A} \_$HOM and $\mathrm{V} \_\mathrm{HSM}$ and A_HOM conditions using unpaired-samples $t$-tests, the onset latency of self-motion was not significantly different regardless
} 

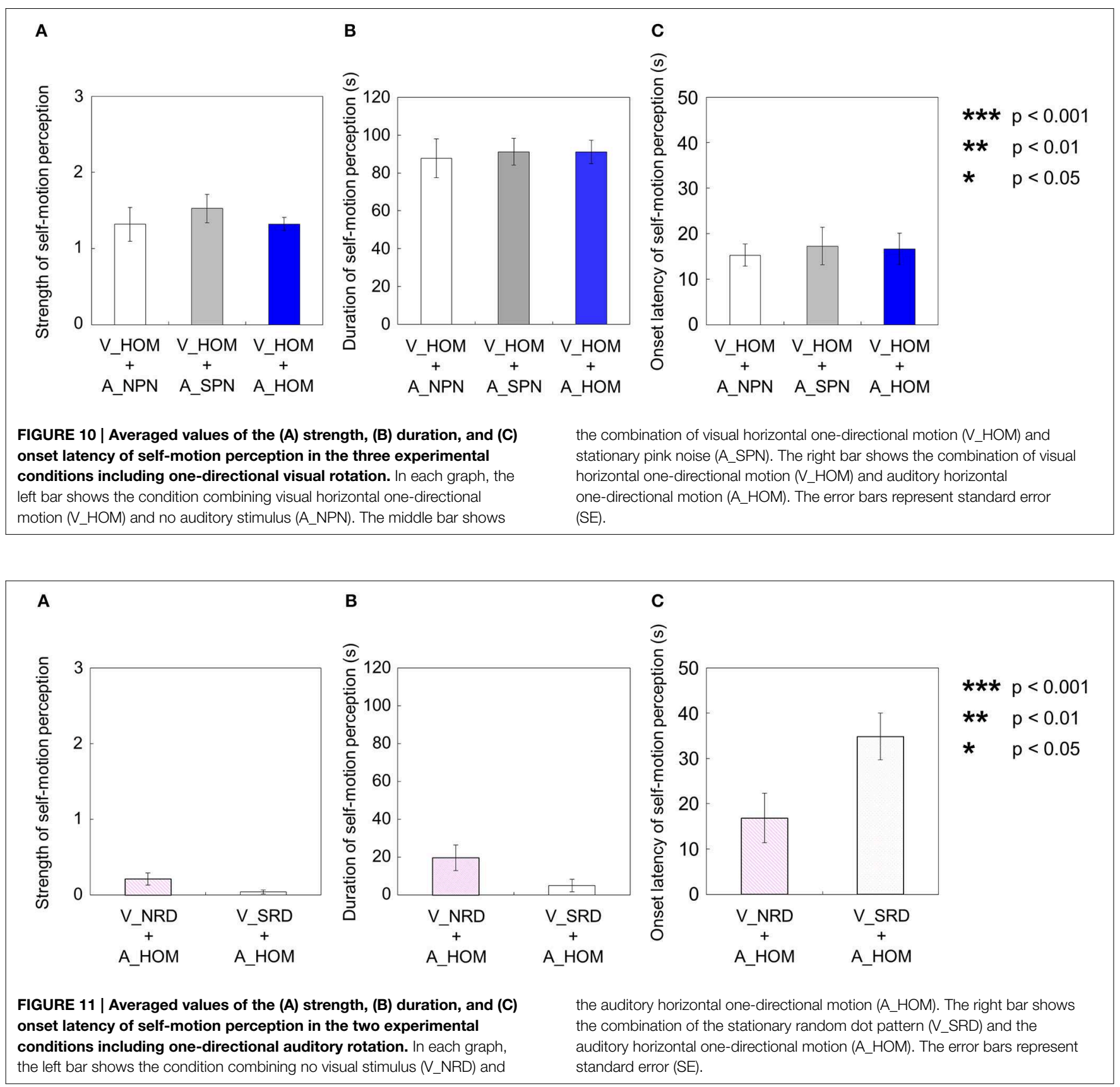

\section{When both Visual and Auditory Stimuli Moved in One Direction}

When both the visual and auditory stimuli of horizontal onedirectional motion were presented (V_HOM + A_HOM), all participants predominantly perceived self-motion in the opposite direction of the visual rotation stimulus (39 out of 40 trials). The strength, duration, and onset latency of self-motion were not significantly different regardless of the consistency of rotational direction between visual and auditory stimuli [strength: $t_{(9)}=$

of whether self-motion perception was induced by the above two experimental conditions $[t(13)=0.74, p=0.47, d=0.44]$.
$0.28, p=0.79, d=0.065$; duration: $t_{(9)}=1.32, p=0.22$, $d=0.083$; onset latency: $t_{(9)}=0.30, p=0.77, d=0.080$; Figures 11A-C].

We compared each of the three values-strength, duration, and onset latency-of self-motion perception across the three different experimental conditions (V_HOM + A_NPN vs. V_HOM + A_SPN vs. V_HOM + A_HOM). To do this, we averaged amount of time that the participants experienced selfmotion in the opposite direction of visual/auditory motion and the mean strength of perceived self-motion during the time, and averaged each of the above values across trials by visual information. We found no significant differences in those values 


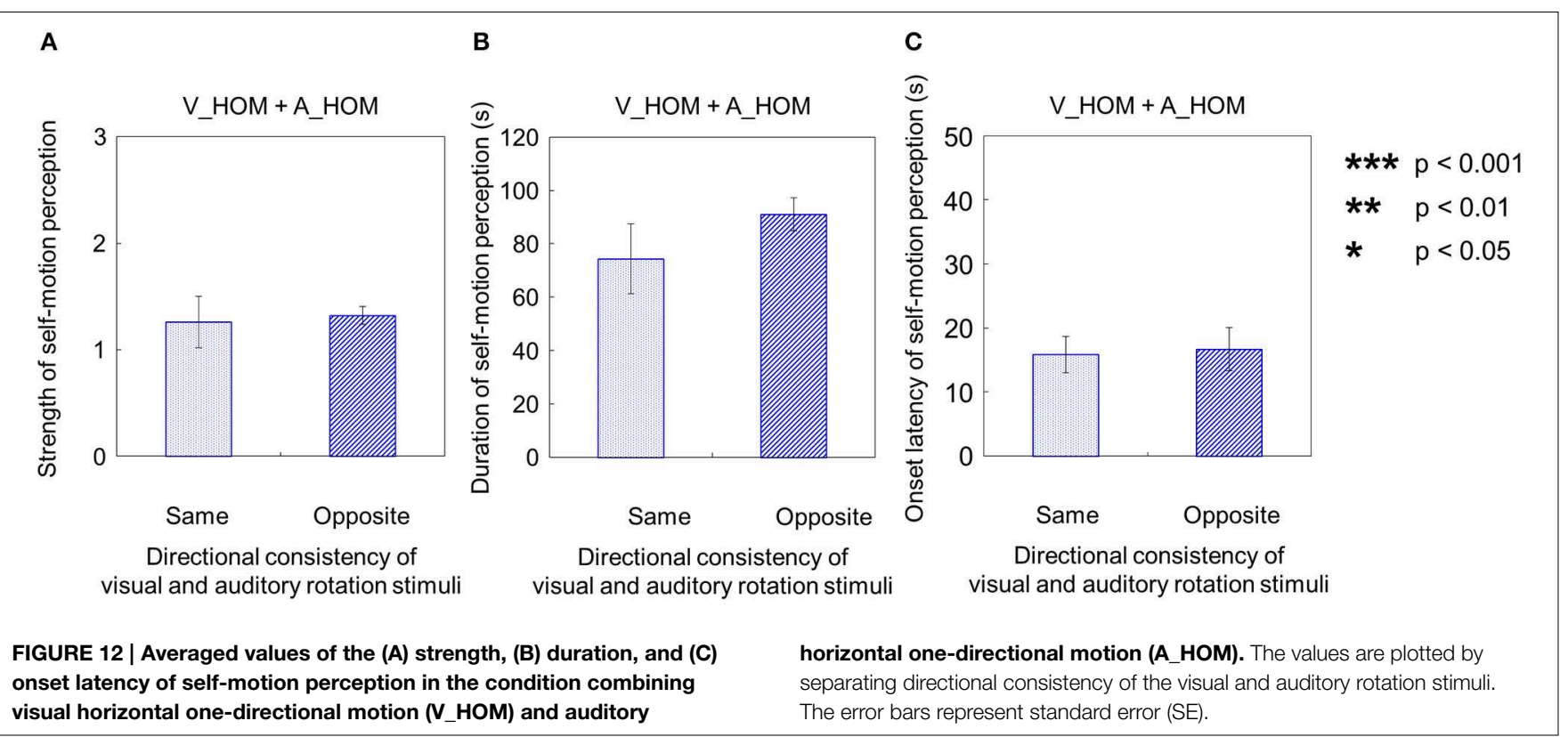

among the three experimental conditions using a One-Way repeated measures ANOVA [strength: $F_{(2,27)}=0.41, p=0.67$, $\widehat{\eta}^{2}=0.029$; duration: $F_{(2,27)}=0.82, p=0.45, \widehat{\eta}^{2}=0.057$; onset latency: $F_{(2,27)}=0.85 \times 10^{-2}, p=0.92, \widehat{\eta}^{2}=0.0062$; Figures 12A-C].

\section{Discussion}

This study indicated that auditory motion information determined the perceived direction of self-motion during simultaneous presentation of visual and auditory motion information, at least when visual stimuli moved in opposing direction (around the yaw-axis). Moreover, our results indicated that auditory motion information can be enhanced to contribute to self-motion perception when presented with a visual motion stimulus, as the strength and duration of self-motion perception with auditory motion information alone (in the V_NRD + A_HOM condition) was weaker and shorter than those of auditory motion information presented with visual shearing motion (in the V_HSM + A_HOM condition).

Previous studies reported that auditory motion information facilitates self-motion perception when the directions of visual and auditory motion stimuli are identical (Riecke et al., 2009; Seno et al., 2012; Keshavarz et al., 2014). However, those studies also indicated that auditory motion information is weaker than visual motion information in producing selfmotion perception. When the directions of visual and auditory motion information conflict with each other, visual information dominates self-motion perception. The strength and direction of perceived self-motion are the same as those when visual motion information is presented alone (Seno et al., 2012).

Our results also indicated that auditory motion information is weaker than visual information for producing self-motion perception in two respects: (1) visual information dominated self-motion perception when visual and auditory motion information were simultaneously presented in conflicting directions, and (2) self-motion perception produced by auditory motion information alone was weaker than was that produced by visual motion information alone. Item (1) was confirmed based on two different results. First, the direction of selfmotion perception was determined by visual information when simultaneously presented visual and auditory motion directions conflicted with each other. Second, the strength, duration, and onset latency of self-motion perception induced by simultaneous visual and auditory yaw motion information, conflicting with each other in terms of rotational direction, were not significantly different from those obtained by visual yaw motion alone. This result was the same as that of Seno et al. (2012), who used different types of motion from those employed in the present study. Using radially expanding visual motion and weakening pure tone sounds, Seno et al. (2012) indicated that self-motion perception was neither inhibited nor enhanced when the directions of visual and auditory stimulus rotation were opposite.

Item (2) was also confirmed by two different findings. First, the strength and duration of self-motion perception produced by visual motion information alone were significantly stronger and longer than were those produced by auditory stimuli alone, while the onset latencies were not significantly different from each other. This finding would be further supported by the results of comparing between the V_HOM +A_SPN and V_SRD + A_HOM conditions. Second, self-motion perception induced by auditory motion information alone was not strong enough to persist. Five of the 10 participants perceived self-motion with auditory motion information alone. This ratio is consistent with previous reports, which range from 20 to $60 \%$, depending on the auditory motion stimulus used (Lackner, 1977; Larsson et al., 2004). Moreover, auditory one-directional motion stimuli did 


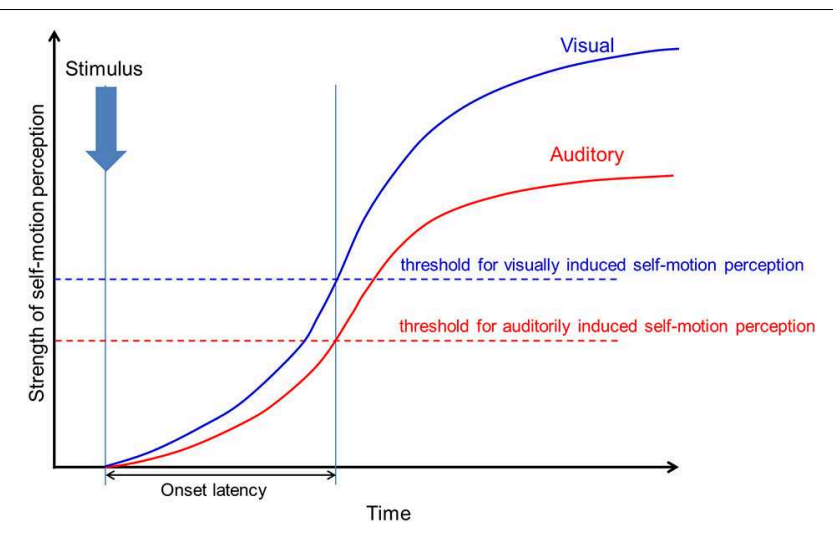

FIGURE 13 | The thresholds of visually and auditorily induced self-motion perception. The blue line shows the strength threshold of visually induced self-motion perception. The red line shows the strength threshold of auditorily induced self-motion perception.

not consistently induce self-motion opposite to the direction simulated by the stimulus. This is consistent with Sakamoto et al. (2004), who used auditorily linear motion stimuli. This finding might be explained by participants incorrectly perceiving the rotational direction simulated by the auditory stimulus. In fact, the literature suggests that perceived rotational direction of auditory stimuli does not necessarily coincide with that of the actual stimulus, probably because of front-back confusion (Young, 1931; Wallach, 1939, 1940). Although we confirmed that participants perceived the direction correctly before the experimental trials began, we cannot be certain of the perceived direction of the auditory stimulus itself.

Our results did not indicate that auditory motion information facilitates self-motion perception when the directions of visual and auditory motion are identical, which is inconsistent with previous reports (Riecke et al., 2009; Seno et al., 2012). This difference might be attributable to differences in the plausibility and information balance of visual and auditory stimuli. First, Riecke et al. (2009) used visual stimuli of actual recorded scenes, including a fountain located at a marketplace and a corresponding auditory stimulus. This kind of auditory stimulus can easily induce self-motion perception, as the sound sources typically associated with stationary objects are more effective in triggering auditory self-motion perception than are sounds stemming from moving objects or artificial sounds like pink noise (Larsson et al., 2004; Riecke et al., 2005). Second, the predominance of visual motion information over auditory motion information can vary by the size of the visual field of view (FOV). Riecke et al. (2009) manipulated the size of the visual FOV presented simultaneously with auditory information, and found that auditory information more effectively facilitated self-motion perception with medium-sized FOV $\left(20 \times 15^{\circ}\right)$ than with a large-sized FOV $\left(54 \times 45^{\circ}\right)$. In contrast to Riecke et al. (2009), we used an artificial sound (i.e., pink noise) as an auditory stimulus and a visual stimulus with a diameter of about $94^{\circ}$, which was larger than the size used by Riecke et al. (2009). This might explain why auditory motion information was not as effective in facilitating self-motion perception during simultaneous presentation of visual and auditory information.

If the plausibility and information balance of visual and auditory stimuli varied the extent of auditory facilitation of self-motion, these factors can also vary the predominance of visual over auditory information for the perceived direction of self-motion. In our experimental conditions, auditory motion information was weaker than visual information. It was in the condition in which visual information did not specify self-motion direction that auditory motion information could determine the perceived direction of self-motion. If we could appropriately manipulate the predominance of visual over auditory motion information, auditory motion information might contribute more to determining the perceived direction of self-motion during simultaneous presentation of visual and auditory motion information.

Our results showed no significant difference in the onset latency of visually and auditorily induced self-motion perception, while the perceived strength and duration were significantly different. This discrepancy may be explained by the difference between the strength threshold of visually and auditorily induced self-motion perception, as discussed in Tanahashi et al.'s (2007) simple model of visually induced self-motion perception. Although the strength of auditorily induced perception is weaker than that of visually induced perception, the threshold of auditorily induced perception may be lower than that of visually induced perception, allowing for the onset latency to be equal (Figure 13). This also indicates that weaker self-motion strength can be perceived with auditory information in comparison to visual information, which may be worth examining. At the same time, however, we have to consider that the number of participants in our study was rather small $(N=10)$ and test power was consequently weak $(1-\beta=0.42)$ to detect a relevant effect size (Cohen's $d=0.5$ ). Thus, the non-significant results found in our study need to be treated very carefully.

We conclude that auditory motion information can determine perceived direction of self-motion during simultaneous presentation of visual and auditory motion information, at least when visual stimuli moved in opposing direction (around the yaw-axis). Moreover, considering previous studies (Riecke et al., 2009; Seno et al., 2012), we indicate that auditory information can contribute to self-motion perception when the directions of visual and auditory motion stimuli are identical. The contribution of auditory information seems to depend on the plausibility and information balance of visual and auditory information. 


\section{References}

DeAngelis, G. C., and Angelaki, D. E. (2012). "Visual-vestibular integration for self-motion perception," in The Neural Bases of Multisensory Processes, eds M. M. Murray and M. T. Wallace (Boca Raton, FL: CRC Press), 629-649.

Dichgans, J., and Brandt, T. (1978). "Visual-vestibular interaction: effect on selfmotion and postural control," in Handbook of Sensory Physiology, Vol. 8, eds R. Held, H. W. Leibowitz and H. L. Teuber (Berlin: Springer), 755-804.

Dodge, R. (1923). Thresholds of rotation. J. Exp. Psychol. 6, 107-137. doi: $10.1037 / \mathrm{h} 0076105$

Fisher, M. H., and Kommüler, A. E. (1930). Optokinetisch ausgelöste bewegungswahrnehmungen und optokinetischer nystagmus. J. Psychol. Neurol. 41, 273-308.

Gibson, J. J. (1961). Ecological optics. Vision Res. 1, 253-262. doi: 10.1016/00426989(61)90005-0

Hettinger, L. J., Schmidt, T., Jones, D. L., and Keshavarz, B. (2014). "Illusory selfmotion in virtual environments," in Handbook of Virtual Environments: Design, Implementation, and Applications, 2nd Edn., eds K. S. Hale and K. M. Stanney (Boca Raton, FL: CRC Press), 435-466. doi: 10.1201/b17360-23

Keshavarz, B., Hettinger, L. J., Vena, D., and Campos, J. L. (2014). Combined effects of auditory and visual cues on the perception of vection. Exp. Brain Res. 232, 827-836. doi: 10.1007/s00221-013-3793-9

Kitazaki, M., and Sato, T. (2003). Attentional modulation of self-motion perception. Percept 32, 475-484. doi: 10.1068/p5037

Kleinschmidt, A., Thilo, K. V., Büchel, C., Gresty, M. A., Bronstein, A. M., and Frackowiak, R. S. J. (2002). Neural correlates of visual-motion perception as object- or self-motion. Neuroimage 16, 873-882. doi: 10.1006/nimg.2002.1181

Lackner, J. R. (1977). Induction of illusory self-rotation and nystagmus by a rotating sound-field. Aviat. Space Environ. Med. 48, 129-131.

Larsson, P., Västfjäll, D., and Kleiner, M. (2004). "Perception of self-motion and presence in auditory virtual environments," in Proceedings of the Seventh Annual Workshop of Presence (Valencia), 252-258.

Lepecq, J. C., Giannopulu, I., and Baudonniere, P. M. (1995). Cognitive effects on visual induced body motion in children. Perception 24, 435-449. doi: $10.1068 / \mathrm{p} 240435$

Lishman, J. R., and Lee, D. N. (1973). The autonomy of visual kinaesthesis. Perception 2, 287-294. doi: 10.1068/p020287

Lutfi, R. A., and Wang, W. (1999). Correlational analysis of acoustic orientation cue for the discrimination of auditory motion. J. Acoust. Soc. Am. 106, 919-928. doi: $10.1121 / 1.428033$

Mach, E. (1875). Grundlinien der Lehre von der Bewegungsempfindung. Leipzig: Engelmann

Riecke, B. E., Feureissen, D., and Rieser, J. J. (2008). “Auditory self-motion illusions (circular vection) can be facilitated by vibrations and the potential for actual motion," in Proceedings of the Fifth Symposium on Applied Perception in Graphics and Visualization. (New York, NY), 147-154. doi: 10.1145/1394281.1394309

Riecke, B. E., Schulte-Pelkum, J., Caniard, F., and Bülthoff, H. H. (2005). "Influence of auditory cues on the visually-induced self-motion illusion (circular vection) in virtual reality," in Proceedings of the Eighth Annual Workshop of Presence (New York, NY), 49-57.

Riecke, B. E., Väljamäe, A., and Schulte-Pelkum, J. (2009). Moving sounds enhance the visually-induced self-motion illusion (circular vection) in virtual reality. ACM Trans. Appl. Percept. 6:7. doi: 10.1145/1498700.1498701
Sakamoto, S., Osada, Y., Suzuki, Y., and Gyoba, J. (2004). The effects of linearly moving sound images on self-motion perception. Acoust. Sci Technol. 25, 100-102. doi: 10.1250/ast.25.100

Seno, T., Hasuo, E., Ito, H., and Nakajima, Y. (2012). Perceptually plausible sounds facilitate visually induced self-motion perception (vection). Perception 41, 577-593. doi: 10.1068/p7184

Tanahashi, S., Ujike, H., Kozawa, R., and Ukai, K. (2007). Effect of visually simulated roll motion on vection and postural stabilization. J. Neuroeng. Rehabil. 4:39. doi: 10.1186/1743-0003-4-39

Tanahashi, S., Ujike, H., and Ukai, K. (2012). Visual rotation axis and body position relative to the gravitational direction: effects on circular vection. Iperception 3, 804-819. doi: 10.1068/i0479

Toshima, I., Aoki, S., and Hirahara, T. (2006). Advanced version of a steerable dummy head that tracks human head movement in real time: TeleHead II. J. Acoust. Soc. Jpn. 62, 244-254.

Toshima, I., Aoki, S., and Hirahara, T. (2008). Sound localization using an acoustical telepresence robot: TeleHead II. Presence 17, 365-375. doi: 10.1162 /pres.17.4.392

Urbantschtsch, V. (1897). Über Störungen des Gleichgewichtes und Scheinbewegungen [On disturbances of the equilibrium and illusory motions]. Z. Ohrenheilkd. 31, 234-294.

Väljamäe, A. (2009). Auditory-induced illusory self-motion: a review. Brain Res. Rev. 61, 240-255. doi: 10.1016/j.brainresrev.2009. 07.001

Väljamäe, A., Larsson, P., Västfjäll, D., and Kleiner, M. (2004). “Auditory presence, individualized head-related transfer functions, and illusory ego-motion in virtual environments," in Proceedings of the Seventh Annual Workshop of Presence (Valencia), 141-147.

Wallach, H. (1939). On sound localization. J. Acoust. Soc. Am. 10, 270-274. doi: $10.1121 / 1.1915985$

Wallach, H. (1940). The role of head movements and vestibular and visual cues in sound localization J. Exp. Psychol. 27, 339-368. doi: 10.1037/ h0054629

Warren, R., and Wertheim, A. H. (1990). Perception and Control of Self-Motion. Hillsdale, NJ: Lawrence Erlbaum.

Wood, R. W. (1895). The haunted swing illusion. Psychol. Rev. 2, 277-278. doi: 10.1037/h0073333

Wright, W. G., DiZio, P., and Lackner, J. R. (2006). Perceived self-motion in two visual contexts: dissociable mechanisms underlie perception. J. Vestib. Res. 16, 23-28.

Young, P. T. (1931). The role of head movements in auditory localization. J. Exp. Psychol. 14, 95-124. doi: 10.1037/h0075721

Conflict of Interest Statement: The authors declare that the research was conducted in the absence of any commercial or financial relationships that could be construed as a potential conflict of interest.

Copyright (C) 2015 Tanahashi, Ashihara and Ujike. This is an open-access article distributed under the terms of the Creative Commons Attribution License (CC $B Y)$. The use, distribution or reproduction in other forums is permitted, provided the original author(s) or licensor are credited and that the original publication in this journal is cited, in accordance with accepted academic practice. No use, distribution or reproduction is permitted which does not comply with these terms. 